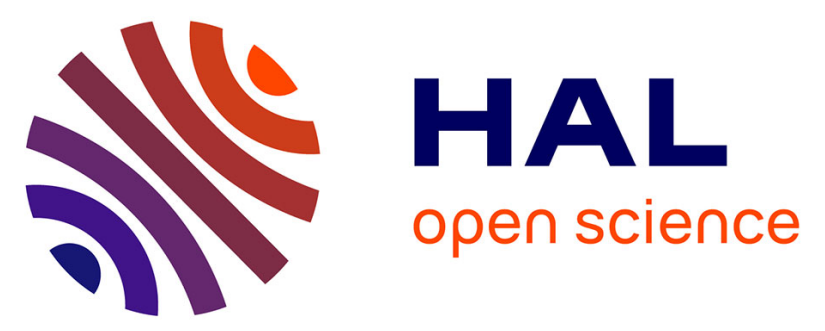

\title{
Unification of compressed imaging techniques in the microwave range and deconvolution strategy
}

Thomas Fromenteze, Ettien Lazare Kpré, Cyril Decroze, David Carsenat, Okan Yurduseven, Mohammadreza Imani, Jonah Gollub, David R. Smith

\section{- To cite this version:}

Thomas Fromenteze, Ettien Lazare Kpré, Cyril Decroze, David Carsenat, Okan Yurduseven, et al.. Unification of compressed imaging techniques in the microwave range and deconvolution strategy. European Microwave Week 2015 - Eurad, Sep 2015, Paris, France. hal-01213874

\section{HAL Id: hal-01213874 \\ https://hal-unilim.archives-ouvertes.fr/hal-01213874}

Submitted on 9 Oct 2015

HAL is a multi-disciplinary open access archive for the deposit and dissemination of scientific research documents, whether they are published or not. The documents may come from teaching and research institutions in France or abroad, or from public or private research centers.
L'archive ouverte pluridisciplinaire HAL, est destinée au dépôt et à la diffusion de documents scientifiques de niveau recherche, publiés ou non, émanant des établissements d'enseignement et de recherche français ou étrangers, des laboratoires publics ou privés. 


\section{Unification of compressed imaging techniques in the microwave range and deconvolution strategy}

\author{
Thomas Fromenteze, Ettien L. Kpré, \\ Cyril Decroze, and David Carsenat \\ Xlim Research Institute, University of Limoges \\ Limoges, France \\ Email: thomas.fromenteze@unilim.fr
}

\author{
Okan Yurduseven, Mohammadreza Imani, \\ Jonah Gollub and David R. Smith \\ Duke University \\ Durham, North Carolina, USA
}

\begin{abstract}
In recent years, several techniques of imaging have emerged based on compressive sensing. Thus, using passive devices able to code and sum the information measured on a radiating aperture, complex UWB beamforming hardwares can be replaced by inverse problems to solve using deconvolution techniques. Two similar compressive techniques have been developed independently, based on a passive multiplexer and on a metamaterial aperture. This paper proposes an unification of these approaches, using a common mathematical formulation and comparing the deconvolution strategy adopted by each research team.
\end{abstract}

\section{INTRODUCTION}

The domain of radar imaging in the microwave range has received a substantial attention in recent years thanks to its application in various domains such as medical diagnosis and concealed objects detection. However, UWB signals and large radiating apertures are required to reach high resolutions in both range and cross-range axis, imposing heavy technical constraints on the active devices required for the generation and reception of waves. In recent years, the development of the compressive sensing theory allowed new imaging techniques to emerge. This new principle is based on the use of a coding aperture, made of a set of radiating elements distributed randomly each with a different resonant frequency. Through experimental characterization of the coded aperture, its complex radiation pattern at each frequency can be predicted, making it possible to reconstruct an image by solving an inverse problem using the aperture's transfer functions and the measured compressed signal at its output. Fig.(1) depicts a generic compressive system.

The waves radiated by an antenna are reflected on a target of spatial reflectivity $M(r)$ and measured by the coding aperture's elements. The received waves are coded depending on the radiating elements' positions and complex weights, corresponding to the compressive device's transfer functions $C\left(r_{R_{X}}, f\right)$ and summed at the unique output. The expression of the measured compressed signal $g(f)$ is, assuming the first Born approximation:

$$
g(f)=\int_{r_{R_{X}}} \int_{r} G\left(r_{T_{X}}, r, f\right) M(r) G\left(r, r_{R_{X}}, f\right) C\left(r_{R_{X}}, f\right) \partial r \partial r_{R_{X}}
$$

with:

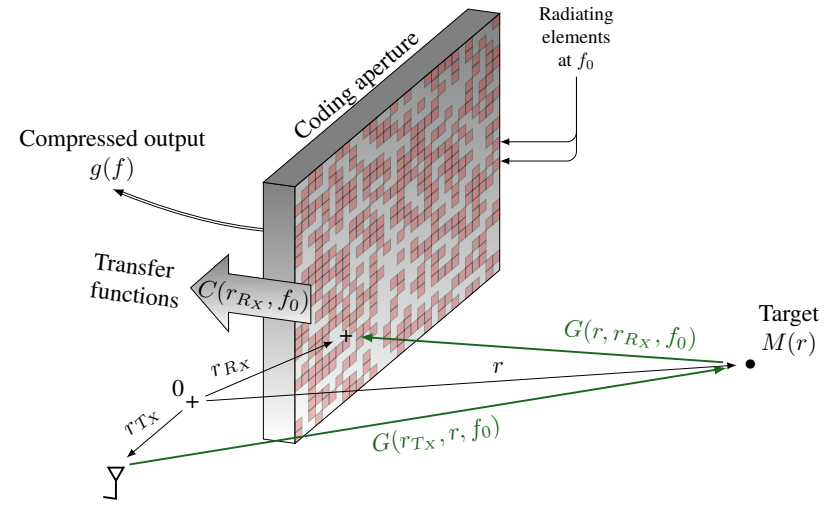

Fig. 1: Imaging system composed of a transmitting antenna and a receiving compressive aperture.

- $r_{R_{X}}$ : the positions of the coding aperture's surface.

- $r_{T_{X}}$ : the position of the transmission antenna.

- $G\left(r_{1}, r_{2}, f\right)$ : the Green function between $r_{1}$ and $r_{2}$.

Thus, the aim of the compressed imaging technique is to compute an estimation of $M(r)$ depending on the measured signal $g(f)$ and on the pre-characterized compressive device's transfer functions. This inverse problem is often confronted to ill-conditioning issues, source of inaccuracy in the reconstruction process. Thus, regularization techniques must be applied to improve the estimation of the spatial reflectivity of the scene.

In the following sections, two techniques that have been developed independently are presented and compared. A first one is based on a passive multiplexer connected to a conventional antenna array while the second one is a metamaterial radiating aperture.

\section{PASSive MUlTiPleXeR}

This technique has been introduced in [1] and is based on a passive multiplexer, which is a 1-to-N ports highly dispersive filter with lowly correlated transfer functions. These properties are reached using the multipath propagation is a closed cavity, able to supply different frequency selectivity depending on the input's port position. Thus, the modal diversity represents here the support of the compression and requires an important volume of cavity. However, compact devices has been introduced 
in [2][3] using 2D chaotic cavities and in [4] with the conception of a 2D photonic crystal. Fig. (2) depicts a compressive device connected to an array of UWB Vivaldi antennas. Waves are radiated by a transmission antenna, reflected by a target and received by the compressive device. Thus, a unique signal is measured for the reconstruction of the scene.

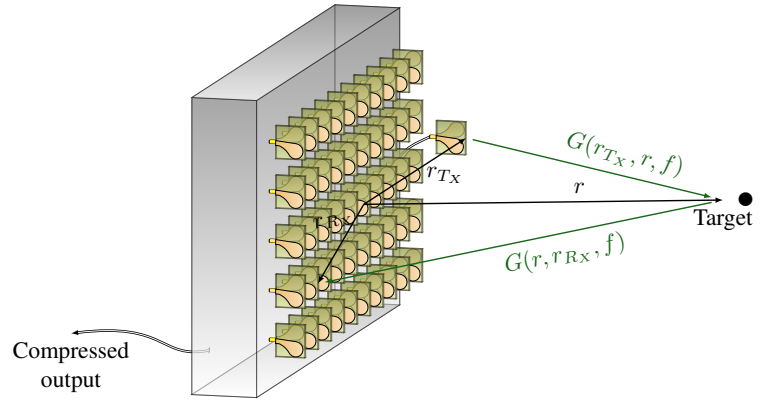

Fig. 2: Illustration of a passive multiplexer connected to an array of UWB Vivaldi antennas.

The characterization of the compressive device's transfer function is performed between the access ports of the antennas and the unique output, using cabled measurement tools. For this technique, the first step is to reconstruct the waveforms received on the compressive device's aperture to make them repropagate in the space. The expression of these reconstructed signals is:

$$
S_{r e c}\left(r_{R_{X}}, f\right)=\left[C\left(r_{R_{X}}, f\right)\right]^{+} g(f)
$$

where $\left[C\left(r_{R_{X}}, f\right)\right]^{+}$is a pseudo-inverse of the matrix $\left[C\left(r_{R_{X}}, f\right)\right]$ computed for each frequency independently. In [1], matched filtering was used, leading to $\left[C\left(r_{R_{X}}, f\right)\right]=$ $\left[C\left(r_{R_{X}}, f\right)\right]^{*}$. This pseudo-inverse corresponds to a simple phase compensation of the device's impact, and is the most stable solution since no magnitude inversion, source of the illconditioning problems, is involved. In [4], the Tikhonov regularization has been adapted to this application by computing the following pseudo-inverse:

$\left[C\left(r_{R_{X}}, f\right)\right]^{+}=\left(\left[C\left(r_{R_{X}}, f\right)\right]^{\dagger}\left[C\left(r_{R_{X}}, f\right)\right]+\beta[I]\right)^{-1}\left[C\left(r_{R_{X}}, f\right)\right]^{\dagger}$

with $\beta$, a positive regularization parameter added to a least-square pseudo-inverse to prevent the inversion of too low values. This computation is performed on a frequency by frequency basis, meaning that the dimension of $\left[C\left(r_{R_{X}}, f\right)\right]$ is $\left(N_{R_{X}} \times 1\right)$ in this calculus.

In [2], the truncated singular value decomposition (tSVD) has been introduced as an alternative to improve the conditioning of the inverse problem. A matrix factorization is used:

$$
\left[C\left(r_{R_{X}}, f\right)\right]=\left[U\left(r_{R_{X}}\right)\right]_{f}\left[\Sigma\left(r_{R_{X}}\right)\right]_{f}\left[V\left(r_{R_{X}}\right)\right]_{f}^{\dagger}
$$

where $\left[U\left(r_{R_{X}}\right)\right]_{f}$ and $\left[V\left(r_{R_{X}}\right)\right]_{f}$ are unitary matrix made of orthonormal basis weighted by the singular values of the quasi-diagonal matrix $\left[\Sigma\left(r_{R_{X}}\right)\right]_{f}\left\{\sigma_{1}, \sigma_{2}, \ldots, \sigma_{N}\right\}$, arranged in descending order. The conditioning is improved by setting the lowest singular values to zero and computing the following pseudo-inverse:

$$
\left[C\left(r_{R_{X}}, f\right)\right]^{+}=\left[V\left(r_{R_{X}}\right)\right]_{f}\left[\Sigma\left(r_{R_{X}}\right)\right]_{f}^{-1}\left[U\left(r_{R_{X}}\right)\right]_{f}^{\dagger}
$$

with $\left[\Sigma\left(r_{R_{X}}\right)\right]_{f}^{-1}$ a quasi-diagonal matrix filled with $\left\{\frac{1}{\sigma_{1}}, \ldots, \frac{1}{\sigma_{\beta}}, 0, \ldots, 0\right\}$. All the remaining singular values must satisfy $\sigma>\beta$, with $\beta$ the regularization parameter. The tSVD and the Tikhonov pseudo-inverse are closely related, the singular value decomposition of the inversed matrix introduced in eq. (3) leads to:

$$
\operatorname{diag}\left(\left[\Sigma\left(r_{R_{X}}\right)\right]_{\text {Tikhonov }}\right)=\left\{\frac{\sigma_{1}}{\sigma_{1}^{2}+\beta}, \frac{\sigma_{2}}{\sigma_{2}^{2}+\beta}, \ldots\right\}
$$

Thus, a similar truncation of the lowest singular values is applied depending on the regularization parameter's value. The main advantage of the tSVD is the possibility to display the useful elements of the linear problem to apply the truncation, but it requires a more expansive computation to perform the matrix factorization at each frequency. Once the signals received by the aperture have been estimated, conventional imaging algorithm can be computed with them to reconstruct an image. The MIMO range migration algorithm introduced in [7] is based on fast Fourier transforms and has been shown efficient, even with 3D reconstructions [8].

\section{METAMATERIAL APERTURE}

An other technique introduced in [5] and developed in [6] uses a radiating aperture made of coupled resonant cELC unit cells able to radiate the required coded patterns depending on the frequency. This metamaterial aperture is associated with a transmission horn matched in the same bandwidth (Fig. 3).

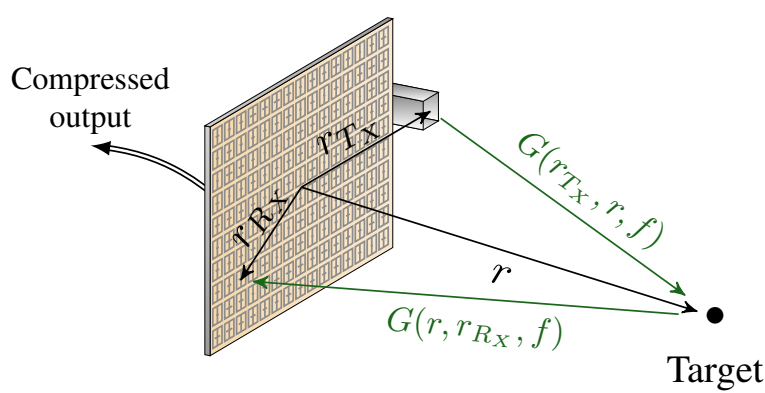

Fig. 3: Metamaterial aperture made of coupled cELC unit cells, associated with a radiating horn.

The study of the compressive device's radiation has been first developed by modeling the field from feeding coaxial probes within parallel-plate waveguide and their couplings to radiating cELC unit cells considered as magnetic dipoles. The measurement of the compressive device's transfer functions can also be performed by a near field scan. A cabled measurement is not possible here since the response of the coupled 
radiating elements is the main source of dispersion, but the near field scan allows for a more accurate measurement of the complete system's radiation. The deconvolution strategy is slightly different from the one presented in the previous section. The radiation of the compressive aperture is discretized over a set of voxels in a region of interest:

$$
[H(r, f)]=\sum_{r_{R_{X}}}\left[G\left(r_{T_{X}}, r, f\right)\right] \cdot\left[G\left(r, r_{R_{X}}, f\right)\right] \cdot\left[C\left(r_{R_{X}}, f\right)\right]
$$

where "." stands for the element-wise multiplication operator. Thus, the reconstructed image $M_{r e c}(r)$ is evaluated in the following way:

$$
M_{r e c}(r)=[H(r, f)]^{+} g(f)
$$

For this technique, several types of pseudo-inverse has also been studied for the computation of $[H(r, f)]^{+}$. In [5], the iterative algorithm TwIST is adapted to this problem using a-priori information on the sparsity of the imaging scene to optimize the estimation. Matched filtering and $l_{2}$-norm leastsquares methods have also been applied for the imaging of continuous targets. This deconvolution method differs from the one presented in the previous section since the complete system's radiation is compensated by the pseudo-inversion, while the previous approach was split in two steps to prevent multiple pseudo-inversions of the compressive device's transfer functions that are independent of the scene to image. Thus, more accurate results can be expected with this second technique, at the cost of a longer computation time. In the following section, a Matlab simulation is presented to compare the two approaches in terms of accuracy and computation time. Thus, a generic compressive device is simulated, associated with an isotropic transmission antenna.

\section{COMPARISON OF THE DECONVOLUTION METHODS}

An aperture made of $30 \times 30$ radiating elements regularly spaced on a $1 \mathrm{~m}^{2}$ square are randomly weighted depending on the frequency is studied in the $2-10 \mathrm{GHz}$ range. The compressive aperture is centered on the $x z$-axis and a transmission antenna is set on the side of the array at the coordinate $(x=-0.6 \mathrm{~m}, z=0 \mathrm{~m})$. For the same scenario, a comparison of point spread functions (PSF) is presented depending on the deconvolution approach. The frequency vector is sampled by 1001 points and the imaging scene is discretized by $90 \times 13 \times 90$ voxels in a volume delimited by $x \in[-0.5,0.5] \mathrm{m}, y \in$ $[0.31,0.76] \mathrm{m}, z \in[-0.5,0.5] \mathrm{m}$. In each simulation, the target is set in the center of the $y$-axis, at $y=0.5 \mathrm{~m}$. Two 3D PSF are presented in Fig. (4) and Fig. (5) using the deconvolution techniques developed for the passive multiplexer and for the metamaterial aperture respectively. For both cases, the reconstructed field is normalized and the $y=0.5 \mathrm{~m}$ plane is extracted to compare the level of sidelobes. This simulation is performed applying the first Born approximation with a punctual target set at $(x=0 \mathrm{~m}, y=0.5 \mathrm{~m}, z=0 \mathrm{~m})$. Both reconstruction are performed using Tikhonov pseudo-inversions with optimized regularization parameters. The results are obtained running Matlab on a computer with a $3.5 \mathrm{GHz}$ 6-core processor and $16 \mathrm{~Gb}$ of RAM.
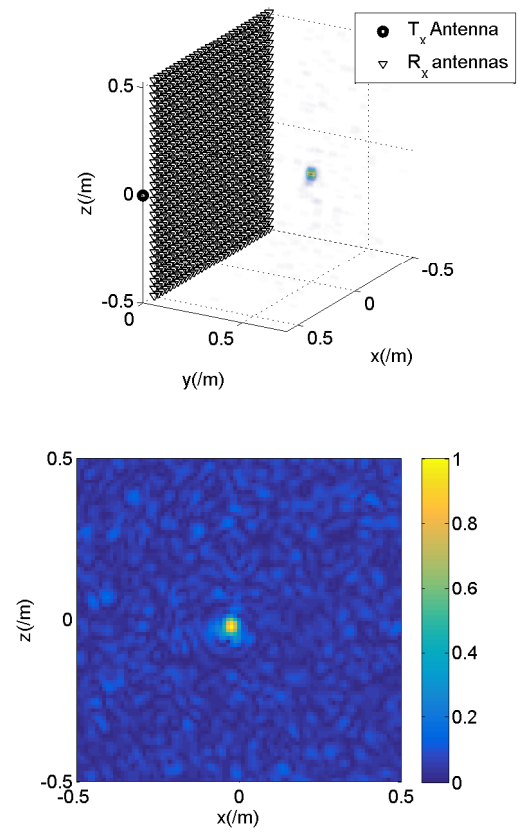

Fig. 4: 3D point spread function for a reconstruction of aperture's field and a range migration algorithm and 2D extraction from the $y=0.5 \mathrm{~m}$ plane
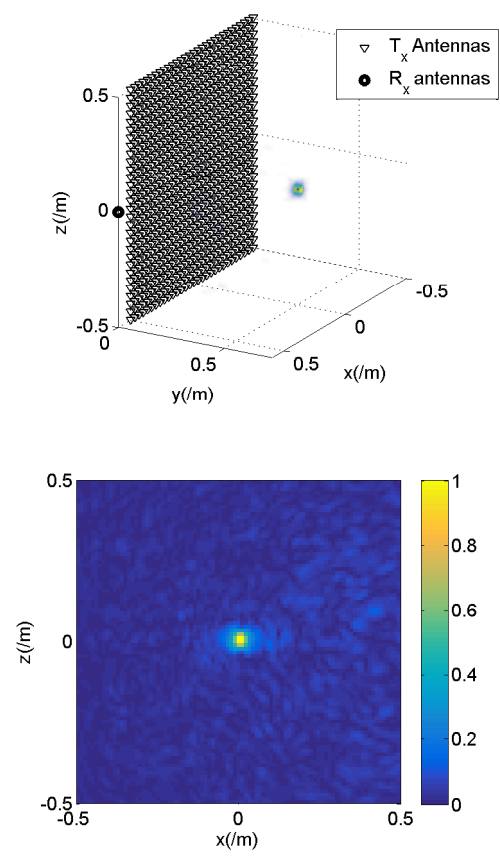

Fig. 5: 3D point spread function for pseudo-inversion of the compressive device radiation discretized on a set of voxels and $2 \mathrm{D}$ extraction from the $y=0.5 \mathrm{~m}$ plane 
The average level of sidelobes is lower in the case of a pseudo-inversion of the radiation compared to the case of a reconstruction of the aperture's field re-propagated with a range migration algorithm. Indeed, the RMA is based on a stationary phase approximation allowing for a reduction of the required computing time at the price of slight distortion of the images. 1D cuts for $x=0 \mathrm{~m}$ and $z=0 \mathrm{~m}$ are extracted from the $y=0.5 \mathrm{~m}$ plane and compared in Fig. (6).

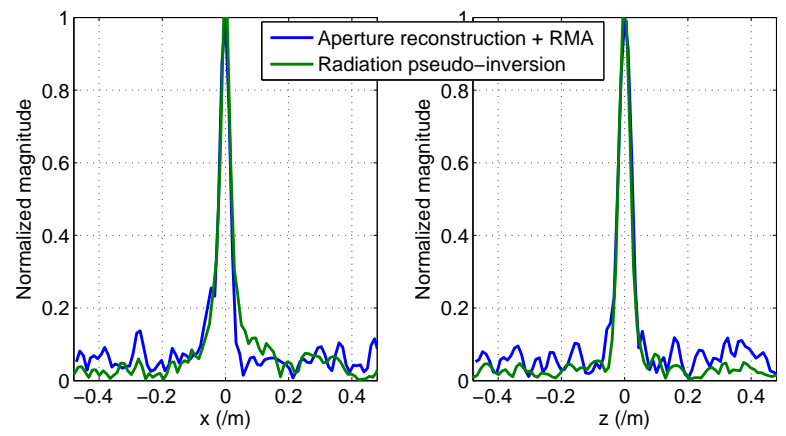

Fig. 6: Comparison of the PSF for 1D cut along the $x$-axis and $z$-axis.

For the studied scenario, the computation time required with each deconvolution method are gathered in table (I). In each case, a part of the algorithm is independent of the imaging scene and is pre-computed.

TABLE I: Comparison of the computation time depending on the chosen deconvolution strategy

\begin{tabular}{|c||c|c|}
\hline & Pre-computation time & Computation time \\
\hline Aperture reconstruction \& RMA & $4.8 \mathrm{~s}$ & $2.4 \mathrm{~s}$ \\
\hline Spatial deconvolution & $38 \mathrm{~min}$ & $66 \mathrm{~s}$ \\
\hline
\end{tabular}

In the case of a spatial deconvolution, a $1.5 \mathrm{~Gb}$ matrix $[H(r, f)]$ is pre-computed for the depicted scenario and saved, working with double-precision floats. The longer computation time for this case is explained by the matrix loading required before the reconstruction of a scene. To conclude, a simulation of a gun shape is presented with the same radiating aperture to show the imaging capacity of this compressive system. The image obtained for conventional digital beamforming using the signals measured from each receiving element is compared with the one obtained with only one compressed signal measured at the output of the imaging device (Fig. 7). For this case, a random matrix of 10001 frequency points has been used, associated with the range migration algorithm. The estimation of the $30 \times 30$ received signals can be performed accurately enough to reconstruct a $3 \mathrm{D}$ gun shape close to the non-compressed case.

\section{CONCLUSION}

In this paper, the similarities between two techniques of compressed imaging in the microwave range have been presented. Although independently developed, this two techniques are basically the same and share a common mathematical formulation. The use of a near field scan seems to be the best way to characterize the imaging system since it allows for
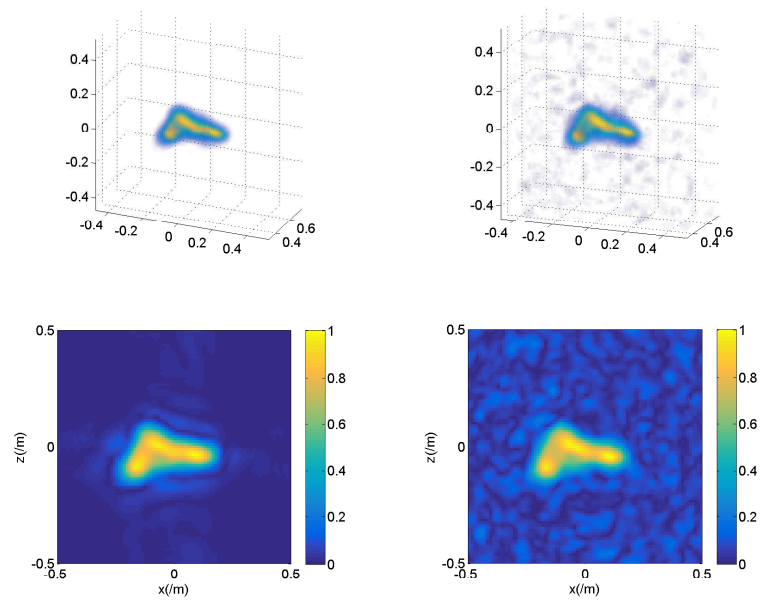

Fig. 7: Imaging of a gun using conventional digital beamforming (left), compared to compressed imaging (right).

an accurate measurement of the radiation patterns, including the inter-element coupling that is not compensated for the case of the passive multiplexer. Furthermore, the use of range migration algorithm should be adapted to the metamaterial apertures when fast imaging is required, subject to a slight loss of accuracy. This new technology represents a promising way to simplify the complex hardware architectures required for high resolution microwave imaging. Future efforts in each research group will be focused on improving the imaging capabilities of these techniques, implementing compressive aperture to aperture imaging [8], GPU acceleration, and polarization imaging.

\section{REFERENCES}

[1] D. Carsenat and C. Decroze, "UWB antennas beamforming using passive time-reversal device”, IEEE Antenn. Wireless Propag. Lett., vol. 11, pp. 779-782, 2012.

[2] T. Fromenteze, C. Decroze and D. Carsenat, Miniaturized Device for Passive Microwave UWB Beamforming, 8th European Conference on Antennas and Propagation, 2014.

[3] T Fromenteze, C Decroze, and D Carsenat, UWB passive beamforming for large antenna arrays, IEEE International Conference on UltraWideBand, 2014.

[4] T. Fromenteze, C. Decroze, and D. Carsenat, "Waveform coding for passive multiplexing: Application to microwave imaging", IEEE Trans. Ant. Prop., vol. 63, no. 2, 2014.

[5] J. Hunt, T. Driscoll, A. Mrozack, G. Lipworth, M. Reynolds, D. Brady, and D.R. Smith, "Metamaterial apertures for computational imaging", Science, vol. 339, pp. 310-313, 2013.

[6] G. Lipworth, A. Mrozack, J. Hunt, D. L. Marks, T. Driscoll, D. Brady, and D.R. Smith, "Metamaterial apertures for coherent computational imaging on the physical layer". JOSA A, vol 30, no 8, pp.1603-1612, 2013.

[7] X. Zhuge and A.G. Yarovoy, "Three-Dimensional Near-Field MIMO Array Imaging Using Range Migration Techniques", Image Processing, IEEE Transactions on, vol.21, no.6, pp. 3026-3033, 2012.

[8] T. Fromenteze, C. Decroze, and D. Carsenat, "Passive UWB multiplexing device for a single-port MIMO RADAR", Microwave Symposium (IMS), 2014 IEEE MTT-S International, 1-6 June 2014. 